\title{
Ethics Vignette
}

\section{Coping With the Oncology Workforce Shortage: Transitioning Oncology Follow-Up Care to Primary Care Providers}

\author{
David Debono, $M D$
}

Division of Hospitalist Medicine, Henry Ford Hospital, Detroit, MI

\section{Vignette}

A 56-year-old woman was diagnosed with stage I breast cancer 10 years ago. Her tumor was $1.9 \mathrm{~cm}$ in size, disease was estrogen and progesterone receptor positive, and 12 lymph nodes were negative for metastatic disease. She received four cycles of adriamycin/cyclophosphamide chemotherapy, followed by tamoxifen for 5 years. She became menopausal and was subsequently prescribed letrozole. She now returns to her oncologist having just completed 5 years of letrozole therapy. She is clinically well and has normal mammography, normal bone density, and a normal physical exam. Her previous follow-up oncology visits precipitated significant anxiety, but since becoming spokeswoman of her local breast cancer support group, she reports that she has been better able to adjust to her diagnosis and is less concerned about recurrence.

Her oncologist notes that she is doing quite well and wonders whether the patient should be discharged from his oncology practice. He understands she remains at a small lifetime risk for breast cancer recurrence or second primary breast cancer. However, he also knows there is medical literature illustrating that primary care providers are as adept at diagnosing cancer recurrence as oncologists. ${ }^{1}$ His practice is also facing increasing demand for new patient visits. He decides to discuss transitioning the patient's follow-up to her primary care physician. $\mathrm{He}$ wonders whether this referral will be in his patient's best interest and how best to communicate this transition to the primary care physician.

\section{Discussion}

ASCO and the Institute of Medicine have both produced important reports in the past 2 years documenting an anticipated severe shortage in medical oncologists and other oncology-specific health care personnel over the next 10 to 20 years. ${ }^{2,3}$ Demand for oncology services is expected to increase as a result of an aging population, with concomitant increases in number of cancer diagnoses and cancer survivors and an expansion of therapeutic options for patients with advanced disease. Forces restricting the supply of oncology practitioners include a high rate of retirement among current oncologists in the coming years and limited plans to increase the number of oncology trainees.

An effective response to this impending shortage will require different actions by governmental bodies, academic cancer center leaders, medical societies like ASCO, and community on- cology providers. Some responses will be short term and reactive to immediate needs, whereas other solutions will require proactive and thoughtful deliberation to satisfy patient care needs and achieve institutional goals. In this brief report, we review key questions associated with transitioning oncology follow-up to primary care providers and identify practice tools that can help oncologists facilitate these transitions.

Key question 1. What is the evidence that primary care providers are willing and able to care for the follow-up needs of my patients?

There have been a number of randomized trials assessing breast cancer follow-up performed by primary care providers versus oncologists. In the largest such trial, conducted by Grunfeld et al, ${ }^{1}$ patients with breast cancer in remission were randomly assigned to follow-up with either their primary care providers or oncologists. The authors found that both groups of patients had equivalent numbers of serious clinical events at recurrence (ie, primary care providers detected serious recurrence events at the same rate as oncologists), similar healthrelated quality of life scores, and similar levels of anxiety. This study demonstrated that primary care providers could safely provide breast cancer follow-up.

There are also survey data assessing the views of primary care practitioners on providing follow-up care for cancer survivors. Cheung et $\mathrm{al}^{4}$ surveyed oncology patients, their primary care providers, and their oncologists. The researchers asked survey participants which treating physician was expected to provide primary cancer follow-up, unrelated cancer screenings, and general health care maintenance. The study demonstrated that both primary care providers and oncologists expected to be the lead physician in primary cancer follow-up, illustrating some conflict between the perceptions of the two key physician groups. Their data, however, clearly demonstrated that primary care providers expected to play a significant role in cancer follow-up, a finding that has been supported by others. ${ }^{5}$

Key question 2. Do oncology patients want to have long-term follow-up delivered by primary care providers?

Many oncologists have experienced a patient requesting that follow-up continue at the oncology office rather than transition back to the family physician. These encounters may make oncologists reluctant to refer patients back to their primary care physicians. The survey data reported by Cheung et $\mathrm{al}^{4}$ show 
$91 \%$ of cancer survivors expected their oncologists to provide all or a lot of the required follow-up care for cancer recurrence. This is in contrast to only 33\% of cancer survivors expecting their primary care physicians to provide all or a lot of cancer recurrence follow-up care. The patients in the study had survived 2 years from diagnosis; thus, what patients' expectations might be at a later time point (as in the vignette) could not be assessed.

In their large randomized trial of breast cancer survivors, Grunfeld et al ${ }^{1}$ approached 1,768 patients who had completed initial therapy and asked them to be randomly selected for follow-up with their oncologists or primary care providers. Although the majority of patients agreed to the random assignment (55\% of patients), $45 \%$ of women refused enrollment. It is not known why these women refused to participate; however, it may reflect that oncology patients expect their oncologists to be involved in cancer recurrence surveillance, as noted in the study by Cheung et al. ${ }^{4}$

These data seem to suggest that a hybrid model in which oncologists and primary care providers share follow-up responsibilities (at least for the first several years of follow-up) might satisfy the expectations of all parties. To my knowledge, this specific question of shared follow-up has not been definitively investigated in oncology. However, shared care interventions in chronic disease management have been reviewed, ${ }^{6}$ and primary physical and psychosocial end points did not improve with shared care between specialists and primary care providers, although the frequency of recommended preventive testing prescription improved with shared management.

Key question 3. When I feel a patient is stable, how can I facilitate an effective transition back to the primary care physician?

The move from oncology to primary care follow-up requires the oncologist to fully inform the patient of the safety of and reasons for the transition. This will require clear and compassionate communication techniques to empathically address the anxiety that this transition might entail for the patient. To facilitate the transition and enhance education of patients and primary care physicians, it may be helpful for oncologists to develop a survivorship plan for each patient (particularly for those transitioning back to their primary care physicians). ${ }^{7}$

A survivorship plan might entail two documents provided at different times: first, a treatment summary that describes staging and treatment at the end of prescribed therapy, and second, a survivorship document that outlines long-term expectations and recommendations for the patient and primary care physician. Provision of a survivorship plan might be at the time of transition to long-term follow-up with the primary care provider. Ganz et $\mathrm{al}^{8}$ and Earle ${ }^{9}$ have both reviewed effective survivorship plans and offered recommendations on what components are essential. ASCO is now providing treatment summary templates on its Web site ${ }^{10}$ to help accomplish this important task and is encouraging electronic medical record vendors to incorporate these templates into their products. ${ }^{8}$
Key question 4. What are the basic components of a treatment summary and survivorship plan?

The basic information that a primary care physician and patient should receive in the form of a treatment summary includes type of cancer, staging information, therapy delivered, and some discussion of prognosis. In their review of breast cancer survivorship plans, Ganz et $\mathrm{al}^{8}$ note that key components of survivorship plans include toxicities of therapy (past, current, and possible late toxicities), psychosocial and supportive care needs of the patient and family, surveillance needs for recurrence and new primary cancers, healthy lifestyle changes that could alter the course of disease, and any genetic screening recommended for the patient and family members.

It is clear from the reviews by Ganz et $\mathrm{al}^{8}$ and Earle ${ }^{9}$ that comprehensive survivorship plans can be quite lengthy, and routine execution of these survivorship plans by oncologists will require careful consideration of their local community cultures and of how communication with primary care providers is routinely performed. In her commentary on the provision of cancer follow-up by primary care physicians, Nekhlyudov ${ }^{11}$ notes that both primary care providers and oncologists contribute important elements of longitudinal care for patients with cancer. However, her advice to oncologists who are transitioning patients to primary care physicians with survivorship plans is to "keep it simple." This will be a delicate challenge for oncologists: to synthesize relevant survivorship research, clearly communicate the key elements to patients, and succinctly summarize recommendations for primary care physicians.

\section{Summary}

The anticipated workforce shortage in medical oncology and burgeoning numbers of cancer survivors will challenge medical oncologists to consider transitioning well cancer survivors back to their primary care physicians. This process of transition is likely to be most effective and efficient if oncologists proactively establish plans for survivorship care that satisfy current national standards and are appropriate for the medical cultures of the communities in which they practice. Key take-home points are as follows:

- Survey literature has demonstrated that primary care physicians expect to participate in the longitudinal follow-up of their patients diagnosed with cancer.

- There are high-quality data demonstrating that primary care providers can safely provide follow-up care for patients with cancer.

- A hybrid model in which oncologists and primary care providers share follow-up responsibilities (at least for the first several years of follow-up) might satisfy expectations of all parties.

- Treatment summaries and survivorship plans can be effective ways of clearly communicating key elements of cancer diagnosis and treatment to patients and primary care providers.

- Survivorship plans, which include more components than treatment summaries, will likely become a key feature of oncologic care in the future, as an increasing number of 
well cancer survivors have their follow-up care transitioned to primary physicians.

Accepted for publication on May 19, 2010.

\section{Author's Disclosures of Potential Conflicts of Interest}

The author indicated no potential conflicts of interest.

\section{References}

1. Grunfeld E, Levine MN, Julian JA, et al: Randomized trial of long-term follow-up for early stage breast cancer: A comparison of family physician versus specialist care. J Clin Oncol 24:848-855, 2006

2. Erikson C, Salsberg E, Forte G, et al: Future supply and demand for oncologists: Challenges to assuring access to oncology services. J Oncol Pract 3:79-86, 2007

3. Institute of Medicine and National Cancer Policy Forum of the National Academies: Ensuring Quality Cancer Care Through the Oncology Workforce: Sustaining Care in the 21st Century: Workshop Summary. Washington, DC, National Academies Press, 2009

4. Cheung WY, Neville BA, Cameron DB, et al: Comparisons of patient and physician expectations for cancer survivorship care. J Clin Oncol 27:2489-2495, 2009

5. Del Giudice ME, Grunfeld E, Harvey BJ, et al: Primary care physicians' views of routine follow-up care of cancer survivors. J Clin Oncol 27:3338-3345, 2009

6. Smith SM, Allwright S, O'Dowd T: Effectiveness of shared care across the
Corresponding author: David Debono, MD, Palliative Medicine, Division of Hospitalist Medicine, Henry Ford Hospital, 2799 West Grand Blvd, CFP-1, Detroit, MI 48202; e-mail: ddebono1@hfhs.org.

DOI: 10.1200/JOP.777005

interface between primary and specialty care in chronic disease management. Cochrane Database Syst Rev 3:CD004910, 2007

7. Hewitt J, Greenfield S, Stovall E (eds): From Cancer Patient to Cancer Survivor: Lost in the Transition. Washington DC, National Academies Press, 2005

8. Ganz P, Hahn EE: Implementing a survivorship plan for patients with breast cancer. J Clin Oncol 26:759-767, 2008

9. Earle CC: Failing to plan is planning to fail: Improving the quality of care with survivorship care plans. J Clin Oncol 24:5112-5116, 2006

10. American Society of Clinical Oncology: Chemotherapy treatment plan and summary.http://www.asco.org/ASCOv2/Practice + \&+Guidelines/Quality + Care/ Quality+Measurement+\&+Improvement/Chemotherapy+Treatment+Plan+and+ Summary

11. Nekhlyudov L: "Doc, should I see you or my oncologist?" A primary care perspective on opportunities and challenges in providing comprehensive care for cancer survivors. J Clin Oncol 27: 2424-2426, 2009

\section{The Oncology Electronic Health Record Field Guide}

ASCO has identified the electronic health record (EHR) as an important vehicle for advancing the quality of cancer care and has developed this comprehensive, oncology-specific handbook. The field guide will equip practitioners with the information and resources needed to select and implement current and future oncology-specific EHRs for clinical practice and management as well as quality-of-care measurement and improvement. Order today! 